\title{
MuscleX: A new tool for analyzing X-ray diffraction patterns from muscle and other fibrous systems
}

\author{
Jiranun Jiratrakanvong, Weikang Ma, Gady Agam, and Thomas C. Irving \\ Illinois Institute of Technology
}

X-ray fiber diffraction is the method of choice for determining structural parameters from the sarcomeres in situ in striated muscle. Beamlines at third generation synchrotrons have made it straightforward to collect such information during real physiological experiments in real physiological time potentially generating gigabytes of data in single experimental sessions $\mathrm{A}$ barrier to progress has been the lack of good software tools to analyze the data. Here we describe a new open-source software package, MuscleX, that is optimized for analyzing large sets of X-ray diffraction images from muscle systems. It incorporates some of the functionality of CCP13 but also includes an optimized tool for analyzing equatorial programs from muscle, an important capability especially for the cardiac muscle community. The programs are designed to process entire directories of images with minimal user intervention in order to improve reproducibility, reduce the effects of operator bias and increase efficiency. Analysis tasks that used to take many weeks can now be done in a matter of hours. The package currently consists of "Equator " (analysis of muscle equatorial patterns), Quadrant Folding (average quadrants of a fiber pattern with optional global 2D background subtraction), Projection Traces (1D intensity projections and analysis), Image Merger (averages sequential images to increase signal to noise) and Diffraction Centroids (rapid analysis of spacing changes in along and parallel to the meridional reflections). While the suite was designed primarily with the needs of muscle diffraction in mind, the programs in the suite could be useful for preliminary processing of any kind of fiber diffraction data. We present an overview of the general structure of the software package, describe the specific features of the programs and present some results. We conclude with a discussion of current limitations of MuscleX and suggest some potential future directions. 